\title{
Direct magnetic enhancement of electrocatalytic water oxidation in alkaline media
}

\author{
Felipe A. Garcés-Pineda', Marta Blasco-Ahicart', David Nieto-Castro', Núria López ${ }^{1}$ \\ and José Ramón Galán-Mascarós $\mathbb{1}^{1,2 \star}$
}

Industrially profitable water splitting is one of the great challenges in the development of a viable and sustainable hydrogen economy. Alkaline electrolysers using Earth-abundant catalysts remain the most economically viable route to electrolytic hydrogen, but improved efficiency is desirable. Recently, electron spin polarization was described as a potential way to improve water-splitting catalysis. Here, we report the significant enhancement of alkaline water electrolysis when a moderate magnetic field $(\leq 450 \mathrm{mT})$ is applied to the anode. Current density increments above $100 \%$ (over $100 \mathrm{~mA} \mathrm{~cm}^{-2}$ ) were found for highly magnetic electrocatalysts, such as the mixed oxide $\mathrm{NiZnFe}_{4} \mathrm{O}_{x^{*}}$ Magnetic enhancement works even for decorated Ni-foam electrodes with very high current densities, improving their intrinsic activity by about $40 \%$ to reach over $1 \mathrm{~A} \mathrm{~cm}^{-2}$ at low overpotentials. Thanks to its simplicity, our discovery opens opportunities for implementing magnetic enhancement in water splitting.

W ater electrolysis is widely considered the most promising hydrogen source for the establishment of a clean and sustainable hydrogen economy powered by renewable energy sources ${ }^{1-3}$. In particular, the storage of photovoltaic energy as a fuel via water splitting could represent a dominant contribution to societal energy demands in the near future ${ }^{4}$, combining the excellent efficiency of photovoltaics with the easy storage and transport of fuels. Unfortunately, electrolyser technologies are still too expensive compared with the cheap hydrogen obtained from steam reforming ${ }^{5}$. Because of this, improving these technologies is still a major research challenge, and the subject of several public and private funding schemes.

Nowadays, two electrolyser technologies are commercially available, either based on alkaline liquid electrolytes or polymer electrolyte membranes (PEMs). PEM electrolysers are more efficient and allow for higher production rates (current densities up to $2 \mathrm{~A} \mathrm{~cm}^{-2}$ ), but this comes with the unsolved issue of very expensive parts ${ }^{6}$. This includes the Nafion membrane itself, the titanium metal bipolar plates and the noble metal catalysts $\mathrm{Pt} / \mathrm{C}$ and $\mathrm{IrO}_{2}{ }^{7}$. These noble metals are expensive and extremely scarce, making them a limiting factor for the scale-up of these devices when targeted for mass production. The record for solar to hydrogen efficiency, at an impressive $30 \%{ }^{8}$, was recently reported via the combination of a photovoltaic cell with two PEM electrolysers connected in series. The complete system is very efficient and achieved almost the maximum theoretical limit. However, its viability for large-scale application will require further study since it is based in noble metal catalysts.

In contrast, alkaline liquid electrolysers are a very mature technology, and have been commercially available for over 50 years. In alkaline media, Earth-abundant electrocatalysts are stable enough to run both half-reactions. High-surface-area Ni-based electrodes and catalysts represent the state of the art, providing currents up to $0.5 \mathrm{~A} \mathrm{~cm}^{-2}$ at less than $70 \%$ efficiency ${ }^{9-11}$. Despite its moderate performance, this technology offers the most economic electrolytic hydrogen due to the overall lower cost of its components ${ }^{12}$. However, little room is apparently left for improvement. Benchmarking catalytic studies have demonstrated that many
Earth-abundant metals are able to offer very good performance under alkaline conditions ${ }^{13}$.

A very interesting opportunity to improve water-splitting kinetics comes from the oxygen evolution reaction (OER), which is typically considered the bottleneck for overall water splitting, as a slow and energy-demanding, four-electron process. The formation of the $\mathrm{O}-\mathrm{O}$ bond, on breaking two water molecules must proceed via spin conservation to yield the paramagnetic triplet state of molecular oxygen. Thus, spin polarization of the active catalyst surface may favour parallel spin alignment of oxygen atoms during the reaction to improve the efficiency of the process, as suggested by theoretical studies ${ }^{14,15}$.

The positive effect of spin polarization in OER was recently confirmed ${ }^{16,17}$. Experimental results with chiral catalysts suggested that spin polarization, as induced by the chiral structure of the active centre, was responsible for a superior electrocatalytic activity. In practical terms, the development of chiral OER electrocatalysts is certainly a plausible strategy, but it still represents a clear challenge in the field of affordable/scalable OER catalysts for wide and accessible implementation ${ }^{18}$. Theoretically, it was also proposed that magnetic electrodes may offer analogous positive effects ${ }^{19,20}$. Particularly, the gate effect of spin control for photosystem $\mathrm{II}^{21}$, and generally the effects towards oxygen electrochemistry decomposing the different energy and entropic terms have been suggested ${ }^{14}$.

It is worth mentioning that the use of magnetic fields in water electrolysis has been briefly studied in the past, although from very different perspectives. On the one hand, a positive effect can be found associated with the influence of a magnetic field on the Lorentzian movement for diffusion of reagents and gas bubbles, therefore improving mass transport ${ }^{22-25}$. On the other hand, magnetic improvement has also been achieved by applying high-frequency alternating magnetic fields on a flow cell equipped with electrodes modified with magnetic nanoparticles, in a hyperthermia-like process $^{26}$. In both cases, magnetic fields induce indirect effects on performance, either by improving gas/liquid diffusion or increasing local temperature, respectively. Additionally, both approaches need special electrolyser designs for their convenient exploitation. 

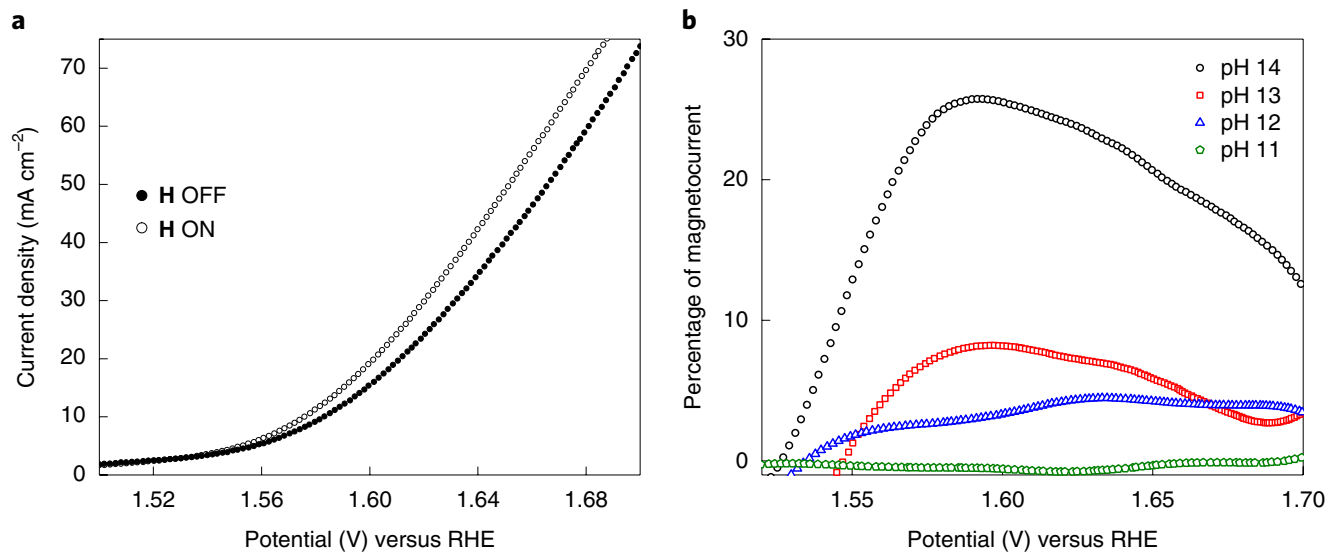

Fig. 1 | Magnetic effect on water electrolysis with a bare Ni-foam anode. a, Polarization curve $\left(5 \mathrm{mV} \mathrm{s}^{-1}\right)$ in $1 \mathrm{M} \mathrm{KOH}(\mathrm{pH} 14)$ with $(\mathrm{ON}$, open circles) and without (OFF, filled circles) an applied $\leq 450 \mathrm{mT}$ magnetic field. $\mathbf{b}$, Magnetocurrent (see equation (4) in Methods) as a function of $\mathrm{pH}$.

Here, we describe how an external magnetic field, easily applied by approaching a permanent magnet, enhances the electrocatalytic activity of magnetic anodes in alkaline conditions. Our experimental data indicate that the magnetic field is affecting the reaction pathway, resulting in a net positive effect, and not related to indirect effects such as those previously described. This magneto-enhancement appears to be proportional to the magnetic nature of the catalysts, and is particularly useful for the highly magnetic iron-nickel oxides, which are the preferred OER catalysts under these conditions.

\section{Magnetic field effect}

We turned our attention to alkaline water electrolysis to investigate the response of a bare $\mathrm{Ni}$-foam anode in a liquid electrolyte $(\mathrm{KOH}$; $1 \mathrm{M}$ ) cell equipped with a platinum mesh cathode and an $\mathrm{Ag} / \mathrm{AgCl}$ 3.5 M KCl reference electrode under an applied magnetic field. This was easily implemented by approaching a commercial neodymium permanent magnet. $\mathrm{Ni}$-foam is not an innocent support because a thin catalytic $\mathrm{Fe}$-doped $\mathrm{Ni}$ oxide layer rapidly evolves on the surface under working conditions, taking up Fe from electrolyte impurities $^{11}$. In our case, after Ni-foam electrode conditioning, the polarization curve (linear sweep voltammetry (LSV)) was measured with and without a magnetic field of $\sim 450 \mathrm{mT}$ (Supplementary Fig. 1 and Methods). The data showed a significant positive effect on spin polarization (Fig. 1a). The onset potential and precatalytic basal current were identical in both regimes. The appearance of a magnetocurrent component above the onset yielded a lower Tafel slope under the applied magnetic field, reaching higher currents at any given potential (Supplementary Fig. 2). The magnetic enhancement, normalized by the basal electrocatalytic performance (equation (4)), reached a maximum $25 \%$ at $\sim 1.60 \mathrm{~V}$ versus the reversible hydrogen electrode (RHE; Fig. 1b). After this threshold, the relative magnetocurrent decreased, which we assigned to diffusion limitations provoked by very intense $\mathrm{O}_{2}$ gas bubbling. In other words, the efficiency of this alkaline electrolysis cell is boosted just by the implementation of a magnetic field (applied from a convenient distance), without any modification of the electrolyser architecture.

The absence of any effect in the precatalytic current indicated that the magnetic field was not affecting the electrode capacitance (related to the total number of redox active sites) or bulk electron transport processes. The different Tafel slope also supported either a different rate-limiting step or an alternative mechanism, suggesting that the magnetic field was speeding up the electrocatalytic reaction kinetics. The genuine mechanistic effect of the spin polarization induced by the permanent magnet in our work was further supported by analogous experiments as a function of $\mathrm{pH}$ (Fig. 1b). The magnetocurrent effect was maximized at $\mathrm{pH} 14$, decreasing as the $\mathrm{pH}$ decreased and becoming negligible below $\mathrm{pH} 11$. An indirect non-mechanistic enhancement, perhaps related to mass transport or local heat, cannot justify such a dramatic $\mathrm{pH}$ dependence. Additionally, this observation suggests that the dominant OER mechanistic pathway changed from alkaline media to neutral conditions ${ }^{27}$, since no magnetic effect is observed below $\mathrm{pH} 11$. This also suggests that the rate-limiting step under neutral conditions was not spin restricted, in contrast with alkaline conditions.

We studied a variety of OER catalysts under identical working conditions: deposited on two-dimensional Ni-foil anodes as Fumatech FAA-3 ionomer inks (see Methods for the detailed procedure). We selected some of the best state-of-the-art OER catalysts (Raney $\mathrm{Ni}, \mathrm{NiFe}_{2} \mathrm{O}_{x}, \mathrm{FeNi}_{4} \mathrm{O}_{x}$ and $\mathrm{Ni}_{2} \mathrm{Cr}_{2} \mathrm{FeO}_{x}^{28-32}$ ) as well as some catalyst $\mathrm{s}^{30,33-35}$ with very different magnetic features (the non-magnetic $\mathrm{IrO}_{2}$, the antiferromagnet $\mathrm{NiO}$, the spinel $\mathrm{ZnFe}_{2} \mathrm{O}_{x}$, and the highly magnetic ferrites $\mathrm{NiZnFe}_{4} \mathrm{O}_{x}$ and $\mathrm{NiZnFeO}_{x}^{3,34,36-40}$ ).

Polarization data (Fig. 2a-i) showed negligible magnetic enhancement for the only non-magnetic catalyst, $\mathrm{IrO}_{2}$, and the appearance of magnetocurrent in all other cases. However, the effect was very different in magnitude, depending on the catalyst. We measured the magnetization curves (Supplementary Fig. 3) for all of these catalysts, looking for a correlation between magnetocurrent and magnetization data. We plotted the magnetocurrent density as a function of bulk magnetization for each catalyst at $450 \mathrm{mT}$ (Supplementary Fig. 4). Given the very different base currents, we did not find any significant trend with absolute values. However, when we analysed the relative magnetocurrent as the percentage of variation in current density (Fig. 3a and Supplementary Table 1), a clear relationship between both parameters was found (Fig. 3b) that appeared to be linear. There was one exception to the general trend: $\mathrm{NiFe}_{2} \mathrm{O}_{x}$ exhibited a lower magnetocurrent than expected from the magnetization data. This may have been due to surface states, since bulk magnetization does not necessarily need to be that of the surface/active sites for all materials.

The maximum relative effect was observed for $\mathrm{NiZnFe}_{4} \mathrm{O}_{x}$, where current doubled from 24 to $40 \mathrm{~mA} \mathrm{~cm}^{-2}$ at $1.65 \mathrm{~V}$ and higher. We modified the working conditions for this set-up to confirm the true magnetic enhancement observed. An identical and consistent magnetocurrent was found when the electrolysis was carried out under a turbulent regime forced by mechanical stirring (Supplementary Fig. 5). This confirmed that the observed magnetic field enhancement was not due to improved mass transport effects, as in multiple previous studies ${ }^{22-25}$. At the same time, the effect was very sensitive to the relative position of the permanent magnet with respect to the electrode, also supporting the directional effect on electrode surface magnetization (Supplementary Fig. 6). 

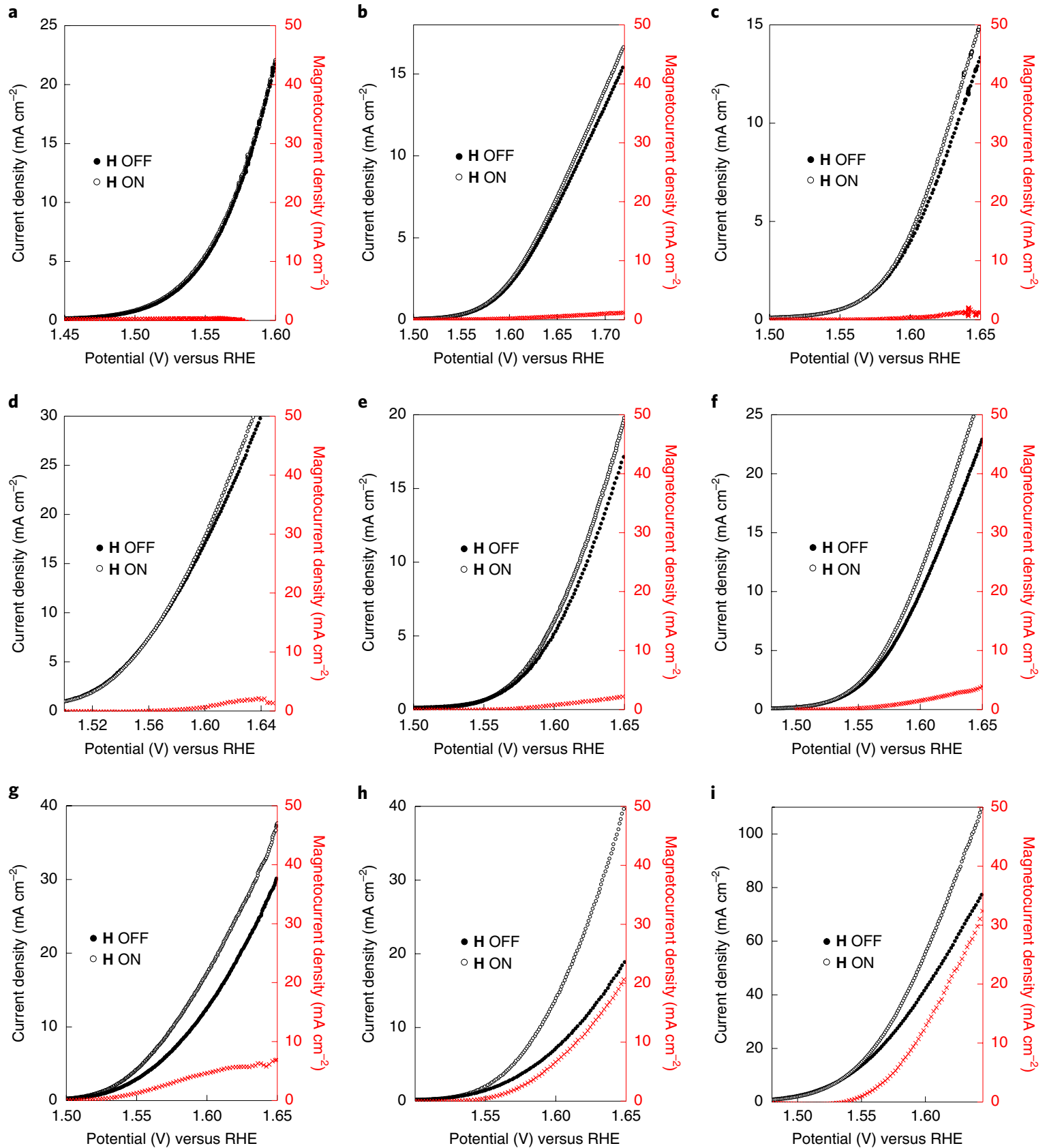

Fig. 2 | Polarization data under an applied magnetic field for different OER catalysts. Polarization curves $\left(5 \mathrm{mV} \mathrm{s}^{-1}\right)$ in $1 \mathrm{M} \mathrm{KOH}$ electrolyte (pH14) for $\mathrm{Ni}$-foil electrodes decorated with OER catalysts (OFF, filled circles), and under an applied $\leq 450 \mathrm{mT}$ magnetic field (ON, open circles). OER catalysts were

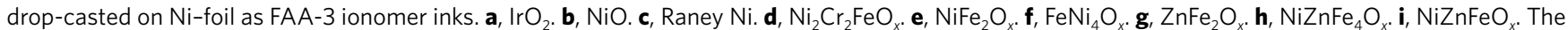
magnetocurrent component (that is, the difference in current density with and without the magnetic field) is represented in red for each catalyst.

$\mathrm{NiZnFe}_{4} \mathrm{O}_{x}$ has another advantage, since it can be magnetically attached to an Ni metal support due to its high magnetization. We decorated an Ni-foam substrate with $\mathrm{NiZnFe}_{4} \mathrm{O}_{x}$ via simple onestep sonication, optimizing the deposition time to $15 \mathrm{~min}$ according to the electrocatalytic performance (Supplementary Fig. 7). The electrode surface was analysed by powder X-ray diffraction, Raman spectroscopy and environmental scanning electron microscopy (Supplementary Figs. 8-10), confirming the presence of the magnetic phase attached to the Ni-foam surface. Excellent longterm stability over $24 \mathrm{~h}$ at $10 \mathrm{~mA} \mathrm{~cm}^{-2}$ was found for these electrodes, despite using exclusively magnetic binding (Supplementary Fig. 11). On application of the magnetic field, the current density roughly doubled (Fig. 3c), reaching over $150 \mathrm{~mA} \mathrm{~cm}^{-2}$ at $1.65 \mathrm{~V}$ versus RHE. We carried out a chronoamperometry experiment by successively moving the magnet next to the electrode (magnet $\mathrm{ON}$ ) and then removing it (magnet OFF). As shown in Fig. 3d, the effect of the magnetic perturbation was immediate, as the current was enhanced consistently under the presence of the magnetic field. Supplementary Videos 1-3 illustrate this effect at basal, $10 \mathrm{~mA} \mathrm{~cm}^{-2}$ and $100 \mathrm{~mA} \mathrm{~cm}^{-2}$ current densities.

Although glass corrosion may occur in alkaline media electrocatalysis ${ }^{41}$, we did not find any effect on reproducibility and stability during our experiments using glassware. The use of Pt mesh counter electrodes in alkaline conditions may also produce migration 


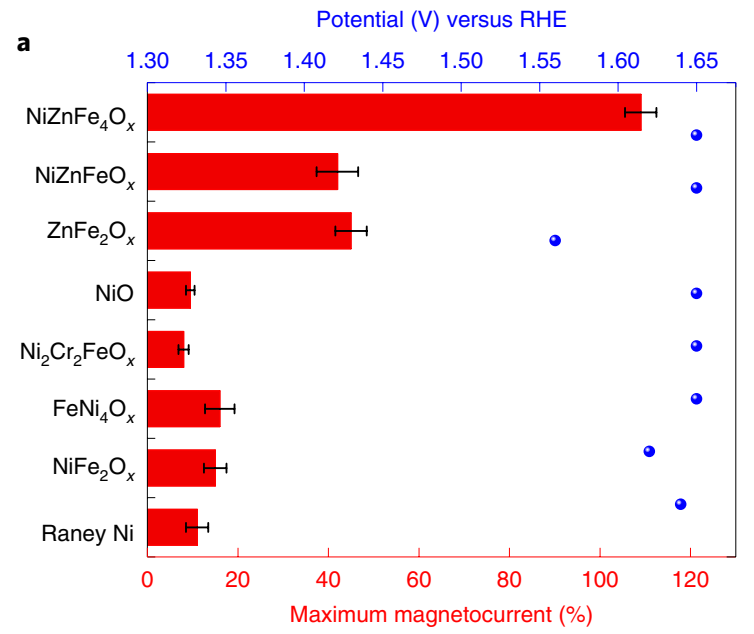

b
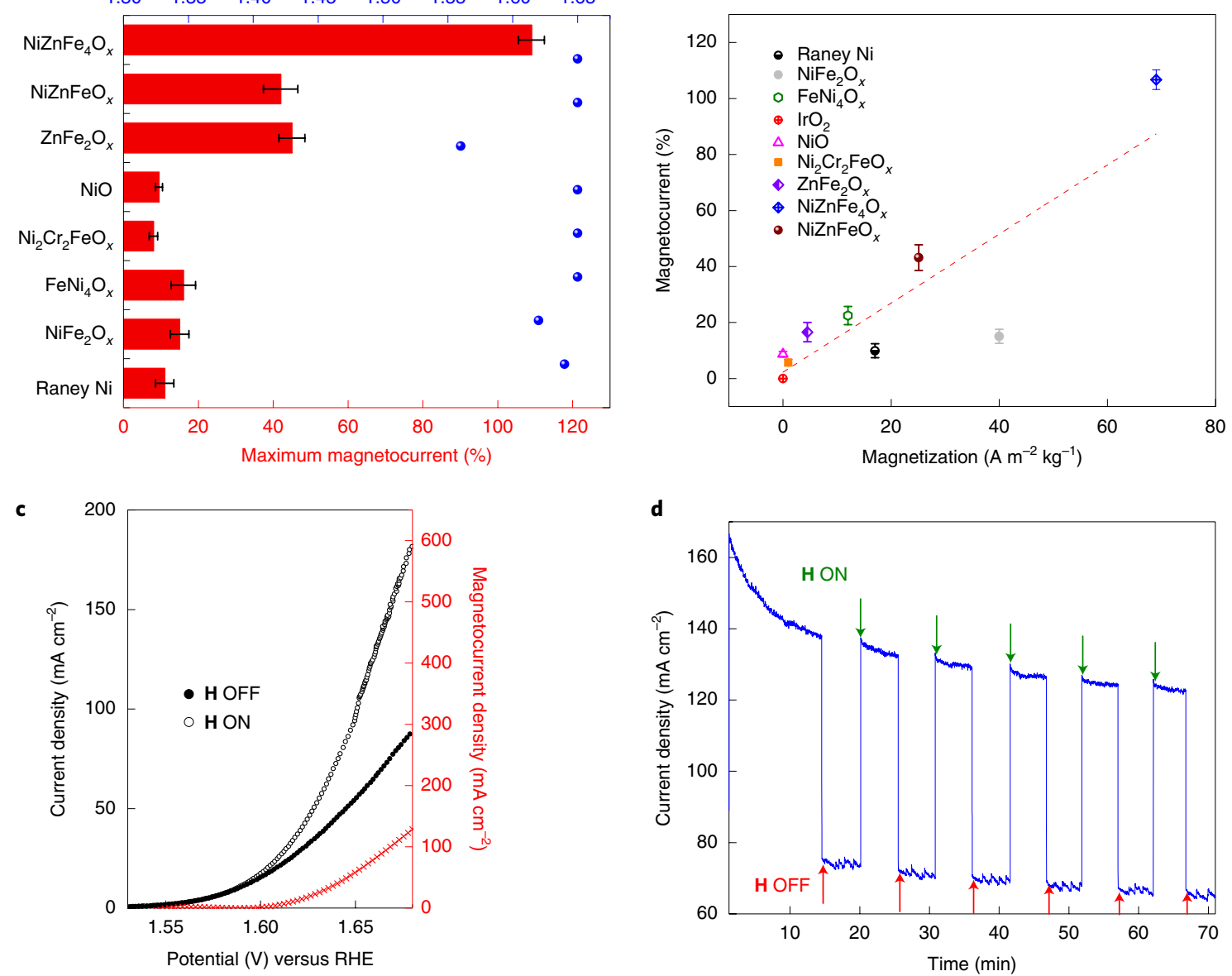

Fig. 3 | Magnetic enhancement of water electrolysis under an applied magnetic field. a, Bar diagram with the maximum magnetocurrent observed for the various magnetic OER catalysts expressed as the relative percentage of the base current, and corresponding applied potential (blue dots). $\mathbf{b}$, Correlation between the maximum relative magnetocurrent (at $1.67 \mathrm{~V}$ versus $\mathrm{RHE}$ ) and bulk magnetization. The dashed line is a guide to illustrate the trend. $\mathbf{c}$, Polarization data $\left(5 \mathrm{mV} \mathrm{s}^{-1}\right)$ for $\mathrm{Ni}$-foam electrodes magnetically decorated (see Methods) with $\mathrm{NiZnFe}_{4} \mathrm{O}_{x}$ particles (OFF, filled circles), and under an applied magnetic field ( $\mathrm{ON}$, open circles). d, A pulsed magneto-chronoamperometry experiment was performed at a constant potential of $\sim 1.67 \mathrm{~V}$ versus $\mathrm{RHE}$ for the Ni-foam electrodes magnetically decorated (see Methods) with $\mathrm{NiZnFe}_{4} \mathrm{O}_{x}$ particles. All data were collected in $1 \mathrm{M} \mathrm{KOH}$ electrolyte (pH14). Error bars represent s.d. $(n=4)$.

and deposition of Pt on other parts of the electrochemical set-up ${ }^{42}$. This is crucial in hydrogen evolution experiments, where Pt is a highly active catalyst, in contrast with OERs, where Pt is considered an inert metal ${ }^{43}$. We repeated the $\mathrm{NiZnFe}_{4} \mathrm{O}_{x}$ key experiments with a carbon counter electrode and a $\mathrm{Hg} / \mathrm{HgO}$ reference electrode (Supplementary Fig. 12). The magnetocurrent results were consistent, independent of counter electrode or reference, and thus confirmed the negligible effect of the Pt mesh counter electrode. Additionally, we repeated the same experiments using a fluorinedoped tin oxide (FTO) glass anode support, to rule out the possible participation of the electrode support. We drop-casted the $\mathrm{NiZnFe}_{4} \mathrm{O}_{x}$ ink on this diamagnetic electrode and observed an even stronger magnetocurrent enhancement, reaching an improvement of $150 \%$ (Supplementary Fig. 13a). This confirmed that the magnetocurrent effect originated at the active sites of the catalysts and was not due to a support effect. Still, the absolute gain in magnetocurrent was significantly higher on the Ni supports (Supplementary Fig. 13b,c), thanks to their metallic character.

The appearance of a magnetocurrent in water oxidation catalysis may be considered analogous to the effect reported for chiral systems. Chiral OER catalysts restrict hydrogen peroxide production ${ }^{17}$ (a significant side reaction at close-to-neutral $\mathrm{pH}$ ), precluding anti- parallel $\mathrm{HO}-\mathrm{OH}$ formation. However, under alkaline conditions, we could not detect the presence of $\mathrm{H}_{2} \mathrm{O}_{2}$ during the OER above pH 11 (Supplementary Fig. 14). This was a major difference between both effects. Apparently, during alkaline water splitting, the $\mathrm{H}_{2} \mathrm{O}_{2}$ side reaction was already minimized, and the applied magnetic field sped up the dominant reaction pathway. Thus, we associate this effect with the spin alignment of oxygen atoms during the reaction, as favoured by the magnetic field (external and local) ${ }^{14}$. Indeed, computational studies suggest that this spin-aligned pathway is thermodynamically favoured in alkaline media (see Supplementary Discussion).

\section{Magnetic enhancement at high current densities}

For technological applications, high geometric current densities are needed. Among the OER catalysts we tested, $\mathrm{NiZnFeO}_{x}$ delivered the highest absolute current densities on magneto-enhancement (Fig. $2 \mathrm{i}$ and Supplementary Fig. 4). To maximize geometric performance, we sprayed nanoparticles of this spinel (Supplementary Fig. 15) onto Ni-foam electrodes as FAA-3 inks. For these electrodes, the magnetic enhancement in the catalytic activity became particularly significant above $100 \mathrm{~mA} \mathrm{~cm}^{-2}$, allowing $20-30 \mathrm{mV}$ savings for a given current, reaching $300 \mathrm{~mA} \mathrm{~cm}^{-2}$ at an overpotential of 

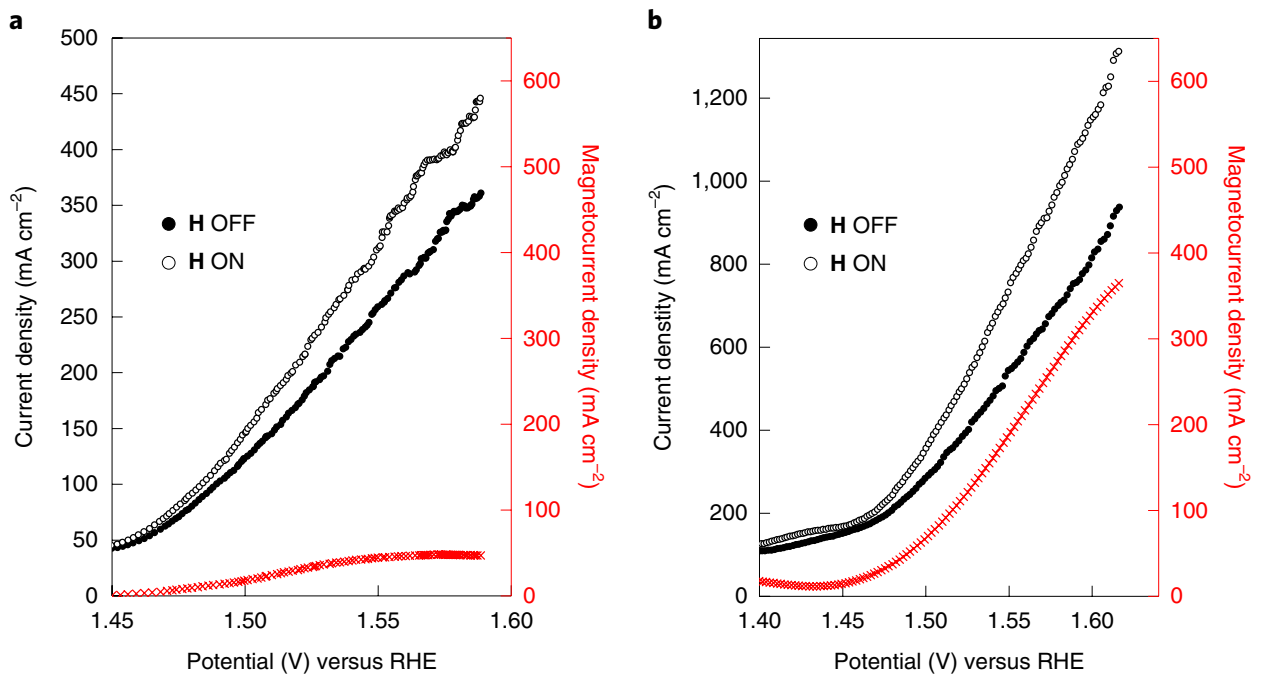

Fig. 4 | Polarization data for surface-modified Ni-foam anodes. a,b, Polarization curves $\left(5 \mathrm{mV} \mathrm{s}^{-1}\right)$ for Ni-foam electrodes decorated with $\mathrm{FeO}{ }_{x}\left(\mathbf{a}\right.$; ref. $\left.{ }^{44}\right)$ and $\mathrm{FeO}_{x} / \mathrm{Ni}_{2} \mathrm{P}$ (b; ref. $\left.{ }^{46}\right)$. Data were collected with (open circles) or without (filled circles) an applied $\leq 450 \mathrm{mT}$ magnetic field. All data were collected in $1 \mathrm{M} \mathrm{KOH}$ electrolyte $(\mathrm{pH} 14)$.

just $341 \mathrm{mV}$ (Supplementary Fig. 16a). These electrodes were robust over $24 \mathrm{~h}$, with and without magnetic enhancement (Supplementary Fig. 16b). Online $\mathrm{O}_{2}$ detection also confirmed quantitative OER Faradaic efficiency with and without the presence of a magnetic field (Supplementary Fig. 17).

Surface-decorated Ni-foam electrodes have been reported with stable and lower overpotentials at very high current densities: those decorated with $\mathrm{FeO}_{x}^{44}$ or $\mathrm{FeO}_{x} / \mathrm{Ni}_{2} \mathrm{P}^{45}$, as grown in situ. We prepared these two electrodes following the published procedures. The electrocatalytic activity was consistent with previous reports (Fig. 4 and Supplementary Table 2). Both electrodes significantly improved their performance under a magnetic field, reducing by $\sim 15 \mathrm{mV}$ the overpotential $(\eta)$ required to reach current densities above $100 \mathrm{~mA} \mathrm{~cm}^{-2}$. Although these potential reductions may seem small, given the very low Tafel slope for these catalysts, the result is an extraordinary current boost, increasing several hundreds of $\mathrm{mA} \mathrm{cm}^{-2}$ just on spin polarization (Supplementary Table 2). For instance, at $1.64 \mathrm{~V}$ versus $\operatorname{RHE}(\eta=415 \mathrm{mV})$, the current density promoted by an $\mathrm{FeO}_{x} / \mathrm{Ni}_{2} \mathrm{P}$-decorated $\mathrm{Ni}$-foam anode increases from 920 to $1,300 \mathrm{~mA} \mathrm{~cm}^{-2}$ under a magnetic field (Fig. 4).

\section{Conclusion}

In summary, we have demonstrated the positive effect of external magnetic fields to speed up electrochemical water oxidation-a key step towards the production of electrolytic hydrogen or other solar fuels. Being a spin-restricted reaction, the magnetic field favours the parallel alignment of oxygen radicals during the formation of the $\mathrm{O}-\mathrm{O}$ bond-the dominant mechanistic pathway in alkaline conditions. We found a trend in the magnetic nature of the catalysts, with a negligible effect for non-magnetic catalysts but maximum enhancement for highly magnetic ones, including the $\mathrm{Ni}-\mathrm{Fe}$ oxide series. For some of these catalysts, current densities doubled just by moving a permanent magnet next to the anodic compartment of the glass cell. This magnetic enhancement also occurred at very high currents, opening interesting possibilities for its implementation in alkaline electrolyser technologies. The low fields that promote magneto-enhancement $(<0.4$ Tesla) can be achieved with low-cost ceramic magnets.

\section{Methods}

All chemicals were commercially available (Sigma-Aldrich) and were used without further purification. The Ni-foil substrate (NI000550; Goodfellow) was of $2.0 \mathrm{~mm}$ thickness and $99.0 \%$ purity. The Ni-foam substrate (NI003852; Goodfellow) was of $1.6 \mathrm{~mm}$ thickness, $95 \%$ porosity and $99.5 \%$ purity. The FTO-coated glass slides were $12-14 \Omega$ per square surface resistivity (NSG TEC; Pilkington; $15 \mathrm{~A} ; 2.2 \mathrm{~mm}$ ). The Nd-magnet ring (IMA) had the following dimensions: diameter, $A=76 \mathrm{~mm}$; internal diameter, $B=35 \mathrm{~mm}$; thickness, $C=6 \mathrm{~mm}$.

Oxide synthesis. The metal oxides $\mathrm{NiO}, \mathrm{NiFe}_{2} \mathrm{O}_{x}, \mathrm{FeNi}_{4} \mathrm{O}_{x}$ and $\mathrm{Ni}_{2} \mathrm{Cr}_{2} \mathrm{FeO}_{x}$ were obtained using a combustion method ${ }^{46}$. A starting aqueous solution $(50 \mathrm{ml})$ of the metal nitrate salts in the desired ratio was prepared, with the iron concentration fixed to $50 \mathrm{mM}$. Glycine was added to the aqueous solution in a glycine-to-metal molar ratio of 1.20 and stirred until total dissolution. Afterwards, the solution was heated to $200^{\circ} \mathrm{C}$ until the solvent was totally evaporated and the glycine had combusted. This flamy combustion process was accompanied by the vigorous emission of gasses $\left(\mathrm{CO}_{2}, \mathrm{~N}_{2}\right.$ and water vapour). The resulting porous dark solids were recovered and sintered at $1,100^{\circ} \mathrm{C}$ in a tubular oven for $1 \mathrm{~h}$ in air. Finally, the sintered materials were mechanically milled in an agate ball mill for $15 \mathrm{~min}$ at $25 \mathrm{~Hz}$ for a final particle size of $\sim 150-250 \mathrm{~nm}$.

The metal oxide $\mathrm{NiZnFe}_{4} \mathrm{O}_{x}$ was obtained via a coprecipitation method. The desired amounts of $\mathrm{NiCl}_{2} \cdot 6 \mathrm{H}_{2} \mathrm{O}(5 \mathrm{mmol}), \mathrm{ZnCl}_{2}(5 \mathrm{mmol})$ and $\mathrm{FeCl}_{3} \cdot 6 \mathrm{H}_{2} \mathrm{O}$ $(20 \mathrm{mmol})$ were dissolved in $100 \mathrm{ml}$ of distilled water. Then, precipitation was induced with $15 \mathrm{mmol}$ of $\mathrm{NaOH}$. The reactive solution was kept at $85^{\circ} \mathrm{C}$ for $1 \mathrm{~h}$. The precipitate was thoroughly washed with distilled water several times to remove sodium and chlorine excess from the solid. Then, the wet solid was dried at $110^{\circ} \mathrm{C}$ overnight, followed by thermal ramp annealing at $550^{\circ} \mathrm{C}$ for $2 \mathrm{~h}$ and $1,000^{\circ} \mathrm{C}$ for $1 \mathrm{~h}$ in a tubular oven. The final product was milled in an agate ball mill for $15 \mathrm{~min}$ at $25 \mathrm{~Hz}$ for a final particle size of $\sim 100-200 \mathrm{~nm}$.

The metal oxide $\mathrm{NiZnFeO}_{x}$ was obtained via a hydrothermal method. Equimolecular amounts of the metal nitrates were dissolved in water (metal concentration: $50 \mathrm{mM}$ ) and the solution was hydrolysed with diluted aqueous ammonia until $\mathrm{pH} 8.5$ was achieved. The solution was introduced to a Teflon cup and mounted in an autoclave at $140^{\circ} \mathrm{C}$ for $2 \mathrm{~h}$. After the hydrothermal treatment, the pressure vessel was cooled in air, the product was washed with $\mathrm{H}_{2} \mathrm{O}$ and $\mathrm{CH}_{3} \mathrm{CH}_{2} \mathrm{OH}$, and the nanoparticles were collected by centrifugation (final particle size: $\sim 8 \mathrm{~nm}$ ).

Electrode preparation. $\mathrm{Ni}$-foil and FTO electrodes. Catalyst inks were prepared following previously reported procedure ${ }^{47}$. The catalyst $(10 \mathrm{mg})$ was mixed with a $10 \mathrm{wt} \%$ of ionomer (FAA-3 ionomer; FUMATEC), and the ink was completed with a liquid $\mathrm{CH}_{3} \mathrm{CH}_{2} \mathrm{OH}: \mathrm{H}_{2} \mathrm{O}$ mixture (3:1 in volume) to a final volume of $1 \mathrm{ml}$. The electrode substrates were cleaned in acetone and Milli-Q water before the ink deposition. Then, $84 \mu \mathrm{l}$ of ink was drop-casted on $1 \mathrm{~cm}^{2}$ of clean Ni-foil or FTO surface. The electrodes were dried at $60^{\circ} \mathrm{C}$ to obtain a total loading of $0.84 \mathrm{mg}$ catalyst $\mathrm{cm}^{-2}$. Blank electrodes were prepared following the same procedure without any catalyst content in the ink formulation.

$\mathrm{Ni}$-foam. $\mathrm{NiZnFe}_{4} \mathrm{O}_{x}$ was deposited on $\mathrm{Ni}$-foam by direct magnetic interaction between the materials. First, Ni-foam was treated with thermal annealing at $450^{\circ} \mathrm{C}$ for $30 \mathrm{~min}$ to generate a passivated layer on the surface. Then, $100 \mathrm{mg}$ of the ferrite was sonicated for $20 \mathrm{~min}$ in $20 \mathrm{ml}$ of Milli-Q water until a homogeneous suspension was obtained. Then, the previously treated $\mathrm{Ni}$-foam was dipped in the suspension 
and sonicated in an ultrasonic bath for different depositions times of 15,30 or $60 \mathrm{~min}$. The final coated $\mathrm{Ni}$-foam electrode was air dried. $\mathrm{NiZnFeO}_{x}$ nanoparticles were deposited on $\mathrm{Ni}$-foam electrodes using the spray technique with analogous FAA-3 ink, as described above. The electrode support was kept at $60^{\circ} \mathrm{C}$ during the spraying process to ensure homogeneous deposition of the catalyst ink.

Electrochemistry. All electrochemical experiments were performed in borosilicate glassware with a Bio-Logic SP-150 potentiostat, $\mathrm{Ag} / \mathrm{AgCl}(3.5 \mathrm{M} \mathrm{KCl})$ reference electrode (ALS) and a Pt mesh counter electrode. Unless otherwise stated, the solution electrolyte used for all electrochemical tests was prepared with $90 \%$ $\mathrm{KOH}$ and Milli-Q water. The desired $\mathrm{pH}$ was measured and adjusted with an 877 Titrino Plus $\mathrm{pH}$ probe (Metrohm). All potentials reported in this manuscript were converted to the RHE reference scale. The exact $\mathrm{pH}$ value was measured for each experiment and used to convert the measured potential to the RHE electrode using the equation:

$$
E_{\mathrm{RHE}}=E_{\mathrm{Ag} / \mathrm{AgCl}}+0.059 \times \mathrm{pH}+0.205
$$

considering $E_{\mathrm{NHE}}^{0}=E_{\mathrm{Ag} / \mathrm{AgCl}}+0.205 \mathrm{~V}$ for our $\mathrm{Ag} / \mathrm{AgCl}(3.5 \mathrm{M} \mathrm{KCl})$ reference electrode.

The water oxidation overpotential $(\eta)$ was calculated by subtracting the thermodynamic water oxidation potential, $E_{\mathrm{O}_{2} / \mathrm{H}_{2} \mathrm{O}}^{0}=1.229(\mathrm{~V})$ versus $\mathrm{RHE}$ $(\mathrm{pH} 14)$, from the experimental potential $\left(E_{\mathrm{RHE}}\right)$ at $\mathrm{pH} 14$ :

$$
\eta=E_{\mathrm{RHE}}-1.229
$$

Linear sweep voltammetry experiments were performed at a $5 \mathrm{mV} \mathrm{s}^{-1}$ scan rate in $1 \mathrm{M} \mathrm{KOH}$. $i R$-compensation was applied to all polarization curves based on resistance data. The magnetic field was applied by moving an $\mathrm{Nd}$-magnet as close as possible to the electrochemical cell ( $\sim 1 \mathrm{~mm}$ glass thickness) (see Supplementary Video 2). The magnetocurrent density was calculated by substracting the LSV values obtained with the applied magnetic field (magnet ON) from the LSV data without the magnetic field (magnet OFF):

$$
\text { Magnetocurrent density }=J\left(\mathbf{H}_{\mathrm{ON}}\right)-J\left(\mathbf{H}_{\mathrm{OFF}}\right)
$$

The magnetocurrent was calculated with the following equation:

$$
\text { Magnetocurrent }(\%)=\frac{J\left(\mathbf{H}_{\mathrm{ON}}\right)-J\left(\mathbf{H}_{\mathrm{OFF}}\right)}{J\left(\mathbf{H}_{\mathrm{OFF}}\right)} \times 100
$$

Oxygen evolution was detected with an Ocean Optics NeoFox oxygen-sensing system equipped with a FOXY probe. The FOXY probe was calibrated with two-point calibration, fixing $0 \% \mathrm{O}_{2}$ under $\mathrm{N}_{2}$ flow and $20.9 \% \mathrm{O}_{2}$ in air. The threeelectrode configuration experiment was performed in a $50 \mathrm{ml}$ two-neck roundbottom flask filled with $1 \mathrm{M} \mathrm{KOH}$ solution $\left(V_{\text {gas space }}=\sim 18 \mathrm{ml}\right)$ with the FOXY probe placed in the gas space. The system was purged with an $\mathrm{N}_{2}$ flow for $30 \mathrm{~min}$ before starting the electrochemical experiment. After removing the $\mathrm{N}_{2}$ flow, a baseline of $6 \mathrm{~min}$ was recorded before starting the chronoamperometry measurement. The moles of $\mathrm{O}_{2}$ generated during the electrochemical experiment were calculated with the following equation, considering ideal gas behaviour:

$$
n_{\mathrm{O}_{2}}=\frac{\% \mathrm{O}_{2} \times P_{\text {total }} \times V_{\text {gas space }}}{R \times T}
$$

where $\% \mathrm{O}_{2}$ is given by the FOXY probe, $P_{\text {total }}$ is $1 \mathrm{~atm}, V_{\text {gas space }}(\mathrm{l})$ is measured for each experiment, $R$ is $0.082 \mathrm{~L}_{\text {atm K}}^{-1} \mathrm{~mol}^{-1}$ and $T$ is $298 \mathrm{~K}$. The Faradaic oxygen production curve was calculated taking into account the charge passed through the system during the chronoamperometry experiment, as described in equation (6):

$$
n_{\mathrm{O}_{2}}=\frac{Q}{n_{\mathrm{e}} \times F}
$$

where $Q(C)$ is the charge passed through the system, $n_{\mathrm{e}}=4$ is the number of electrons needed to generate one molecule of $\mathrm{O}_{2}$, and $F$ is the Faraday constant $\left(96,485 \mathrm{C} \mathrm{mol}^{-1}\right)$.

Hydrogen peroxide was detected by colorimetric titration, with o-tolidine as the redox indicator, following an Elmms-Hauser procedure ${ }^{48}$. An indicator solution was added to $4 \mathrm{ml}$ of the electrolyte solution obtained from the electrochemical cell after $1 \mathrm{~h}$ of chronoamperometry at different potentials, and left to react for $30 \mathrm{~min}$. In the presence of $\mathrm{H}_{2} \mathrm{O}_{2}$, a yellow colour appeared with an ultraviolet-visible absorption peak at around $436 \mathrm{~nm}$. To quantify the $\mathrm{H}_{2} \mathrm{O}_{2}$ concentration, a calibration curve was determined using $30 \% \mathrm{w} / \mathrm{w}$ commercial $\mathrm{H}_{2} \mathrm{O}_{2}$ (Supplementary Fig. 14). $\mathrm{No}_{2} \mathrm{O}_{2}$ was detected in any experiment carried out above $\mathrm{pH} 11$.

Physical methods. Powder X-ray diffraction was obtained with a Bruker AXS D8-Discover diffractometer $(40 \mathrm{kV}$ and $40 \mathrm{~mA})$. Environmental scanning electron microscopy data were obtained using Quanta 600 equipment from FEI under high-vacuum conditions with a Large-Field Detector at $20 \mathrm{kV}$. Transmission electron microscopy was performed with a JEOL JEM1011 microscope operating at 80 or $100 \mathrm{kV}$, equipped with a high-contrast $2 \mathrm{k} \times 2 \mathrm{k}$ AMT mid-mount digital camera. Fourier transform infrared spectroscopy-Raman spectra were collected with a Renishaw inVia Reflex Raman confocal microscope, equipped with a diode laser emitting at $785 \mathrm{~nm}$ at a nominal power of $300 \mathrm{~mW}$, and a Peltier-cooled charge-coupled device detector $\left(-70^{\circ} \mathrm{C}\right)$ coupled to a Leica DM2500 microscope. Calibration was carried out daily by recording the Raman spectrum of an internal Si standard. Rayleigh scattered light was appropriately rejected using edge-type filters. Laser power was used at nominal $1 \%$ to avoid sample damage. Spectra were recorded with the accumulation of at least three scans of $30 \mathrm{~s} \mathrm{each}$. Thermal treatment was carried out using a tubular Nabertherm P330 furnace. The magnetic field calibration of the Nd-magnet was performed using an analogue Hall sensor (HE144; Asensor Technology AB) (Supplementary Fig. 1). The $450 \mathrm{mT}$ quoted in the article corresponds to the maximum magnetic field measured when the Hall sensor was in direct contact with the magnet. During experiments, the magnet was between 1 and $2 \mathrm{~mm}$ from the magnet. Therefore, the magnetic field applied during the experiments was always lower than the maximum limiting value of $450 \mathrm{mT}$. We quote this maximum limit due to the difficulty in estimating the average magnetic field applied to the electrode during operation. The magnetic properties of the bulk materials were determined using an MPMS-XL SQUID magnetometer (Quantum Design) at $300 \mathrm{~K}$.

\section{Data availability}

All experimental data generated or analysed during this study are included in this published article and its Supplementary Information files.

Received: 6 October 2018; Accepted: 30 April 2019;

Published online: 10 June 2019

\section{References}

1. Zhang, B. et al. Homogeneously dispersed multimetal oxygen-evolving catalysts. Science 352, 333-337 (2016).

2. Xu, Y.-F. et al. In situ formation of zinc ferrite modified Al-doped $\mathrm{ZnO}$ nanowire arrays for solar water splitting. J. Mater. Chem. A 4, 5124-5129 (2016).

3. Cady, C. W. et al. Tuning the electrocatalytic water oxidation properties of $\mathrm{AB}_{2} \mathrm{O}_{4}$ spinel nanocrystals: $\mathrm{A}(\mathrm{Li}, \mathrm{Mg}, \mathrm{Zn})$ and $\mathrm{B}(\mathrm{Mn}, \mathrm{Co})$ site variants of $\mathrm{LiMn}_{2} \mathrm{O}_{4}$. ACS Catal. 5, 3403-3410 (2015).

4. McKone, J. R., Lewis, N. S. \& Gray, H. B. Will solar-driven water-splitting devices see the light of day? Chem. Mater. 26, 407-414 (2014).

5. Schmidt, O., Gambhir, A., Staffell, I., Nelson, J. \& Few, S. Future cost and performance of water electrolysis: an expert elicitation study. Int. J. Hydrogen Energy 42, 30470-30492 (2017).

6. Carmo, M., Fritz, D. L., Mergel, J. \& Stolten, D. A comprehensive review on PEM water electrolysis. Int. J. Hydrogen Energy 38, 4901-4934 (2013).

7. Yuan, W., Zhao, M., Yuan, J. \& Li, C. M. Ni foam supported threedimensional vertically aligned and networked layered CoO nanosheet/ graphene hybrid array as a high-performance oxygen evolution electrode. J. Power Sources 319, 159-167 (2016).

8. Jia, J. et al. Solar water splitting by photovoltaic-electrolysis with a solar-tohydrogen efficiency over 30\%. Nat. Commun. 7, 13237 (2016).

9. Song, F. et al. Transition metal oxides as electrocatalysts for the oxygen evolution reaction in alkaline solutions: an application-inspired renaissance. J. Am. Chem. Soc. 140, 7748-7759 (2018).

10. Guo, C. X. \& Li, C. M. Room temperature-formed iron-doped nickel hydroxide on nickel foam as a 3D electrode for low polarized and highcurrent-density oxygen evolution. Chem. Commun. 54, 3262-3265 (2018).

11. Trotochaud, L., Young, S. L., Ranney, J. K. \& Boettcher, S. W. Nickel-iron oxyhydroxide oxygen-evolution electrocatalysts: the role of intentional and incidental iron incorporation. J. Am. Chem. Soc. 136, 6744-6753 (2014).

12. Zeng, K. \& Zhang, D. Recent progress in alkaline water electrolysis for hydrogen production and applications. Prog. Energy Combust. Sci. 36, 307-326 (2010)

13. McCrory, C. C. L., Jung, S., Peters, J. C. \& Jaramillo, T. F. Benchmarking heterogeneous electrocatalysts for the oxygen evolution reaction. J. Am. Chem. Soc. 4, 16977-16987 (2013).

14. Gracia, J. Spin dependent interactions catalyse the oxygen electrochemistry. Phys. Chem. Chem. Phys. 19, 20451-20456 (2017).

15. Gracia, J., Sharpe, R. \& Munarriz, J. Principles determining the activity of magnetic oxides for electron transfer reactions. J. Catal. $\mathbf{3 6 1}$ 331-338 (2018).

16. Mtangi, W., Kiran, V., Fontanesi, C. \& Naaman, R. Role of the electron spin polarization in water splitting. J. Phys. Chem. Lett. 6, 4916-4922 (2015).

17. Mtangi, W. et al. Control of electrons' spin eliminates hydrogen peroxide formation during water splitting. J. Am. Chem. Soc. 139, 2794-2798 (2017).

18. Zhang, W., Banerjee-Ghosh, K., Tassanari, F. \& Naaman, R. Enhanced electrochemical water splitting with chiral molecule-coated $\mathrm{Fe}_{3} \mathrm{O}_{4}$ nanoparticles. ACS Energy Lett. 3, 2308-2313 (2018). 
19. Chretien, S. \& Metiu, H. $\mathrm{O}_{2}$ evolution on a clean partially reduced rutile $\mathrm{TiO}_{2}$ (110) surface and on the same surface precovered with $\mathrm{Au}_{1}$ and $\mathrm{Au}_{2}$ : the importance of spin conservation. J. Chem. Phys. 129, 074705 (2008).

20. Torum, E., Fang, C. M., de Wijs, G. A. \& de Groot, R. A. Role of magnetism in catalysis: $\mathrm{RuO}_{2}$ (110) surface. J. Phys. Chem. C 117, 6353-6357 (2013).

21. Jiao, J., Sharpe, R., Lim, T., Niemantsverdriett, J. W. H. \& Gracia, J. Photosystem II acts as a spin-controlled electron gate during oxygen formation and evolution. J. Am. Chem. Soc. 139, 16604-16608 (2017)

22. Elias, L. \& Hegde, C. Effect of magnetic field on HER of water electrolysis on NiĐW alloy. Electrocatalysis 8, 375-382 (2017).

23. Zheng, Z. et al. Magnetic field-enhanced 4-electron pathway for well-aligned $\mathrm{Co}_{3} \mathrm{O}_{4} /$ electrospun carbon nanofibers in the oxygen reduction reaction. Chem. Sus. Chem. 11, 580-588 (2018).

24. Katz, E., Lioubashevski, O. \& Willner, I. Magnetic field effects on bioelectrocatalytic reactions of surface-confined enzyme systems: enhanced performance of biofuel cells. J. Am. Chem. Soc. 127, 3979-3988 (2005).

25. Monzon, L. M. A., Rode, K., Venkatesan, M. \& Coey, J. Electrosynthesis of iron, cobalt, and zinc microcrystals and magnetic enhancement of the oxygen reduction reaction. Chem. Mater. 24, 3878-3885 (2012).

26. Niether, C. et al. Improved water electrolysis using magnetic heating of FeC-Ni core-shell nanoparticles. Nat. Energy 3, 476-483 (2018).

27. Giordano, L. et al. pH dependence of OER activity of oxides: current and future perspectives. Catal. Today 262, 2-10 (2016).

28. McCrory, C. C. L. et al. Benchmarking hydrogen evolving reaction and oxygen evolving reaction electrocatalysts for solar water splitting devices. J. Am. Chem. Soc. 137, 4347-4357 (2015).

29. Trotochaud, L., Ranney, J. K., Williams, K. N. \& Boettcher, S. W. Solution-cast metal oxide thin film electrocatalysts for oxygen evolution. J. Am. Chem. Soc. 134, 17253-17261 (2012).

30. Gerken, J. B., Shaner, S. E., Massé, R. C., Porubsky, N. J. \& Stahl, S. S. A survey of diverse Earth abundant oxygen evolution electrocatalysts showing enhanced activity from $\mathrm{Ni}-\mathrm{Fe}$ oxides containing a third metal. Energy Environ. Sci. 7, 2376-2382 (2014).

31. Smith, R. D. L. et al. Photochemical route for accessing amorphous metal oxide materials for water oxidation catalysis. Science 340, 60-63 (2013).

32. Seitz, L. C. et al. A highly active and stable $\mathrm{IrO}_{x} / \mathrm{SrIrO}_{3}$ catalyst for the oxygen evolution reaction. Science 353, 1011-1014 (2016).

33. Liu, G., Wang, K., Gao, X., He, D. \& Li, J. Fabrication of mesoporous $\mathrm{NiFe}_{2} \mathrm{O}_{4}$ nanorods as efficient oxygen evolution catalyst for water splitting. Electrochim. Acta 211, 871-878 (2016)

34. Liu, G., Gao, X., Wang, K., He, D. \& Li, J. Uniformly mesoporous $\mathrm{NiO} /$ $\mathrm{NiFe}_{2} \mathrm{O}_{4}$ biphasic nanorods as efficient oxygen evolving catalyst for water splitting. Int. J. Hydrogen Energy 41, 17976-17986 (2016).

35. Dez-Garca, M. I., Lana-Villarreal, T. \& Gómez, R. Study of copper ferrite as a novel photocathode for water reduction: improving its photoactivity by electrochemical pretreatment. ChemSusChem 9, 1504-1512 (2016).

36. Ng, J. W. D. et al. Gold-supported cerium-doped $\mathrm{NiO}_{x}$ catalysts for water oxidation. Nat. Energy 1, 16053 (2016).

37. Islam, M. U. et al. Electrical behaviour of fine particle, co-precipitation prepared Ni-Zn ferrites. Solid State Commun. 130, 353-356 (2004).

38. Wang, X. et al. A novel NiZn ferrite integrated magnetic solenoid inductor with a high quality factor at $0.7-6 \mathrm{GHz}$. AIP Adv. 7, 056606 (2017).

39. He, Q. et al. Electrically controllable spontaneous magnetism in nanoscale mixed phase multiferroics. Nat. Commun. 2, 225 (2011)
40. Su, Y.-Z. et al. One dimensionally spinel $\mathrm{NiCo}_{2} \mathrm{O}_{4}$ nanowire arrays: facile synthesis, water oxidation, and magnetic properties. Electrochim. Acta 174, 1216-1224 (2015)

41. Mayrhofer, K. J. J., Wiberg, G. K. H. \& Arenz, M. Impact of glass corrosion on the electrocatalysis on Pt electrodes in alkaline electrolyte. J. Electrochem. Soc. 155, P1-P5 (2008).

42. Rodriguez, P., Tichelaar, F. D., Koper, M. T. M. \& Yanson, A. I. Cathodic corrosion as a facile and effective method to prepare clean metal alloy nanoparticles. J. Am. Chem. Soc. 133, 17626-17629 (2011).

43. Chen, R. et al. Use of platinum as the counter electrode to study the activity of nonprecious metal catalysts for the hydrogen evolution reaction. ACS Energy Lett. 2, 1070-1075 (2017)

44. Dong, G. et al. In situ formation of highly active NiFe based oxygen-evolving electrocatalysts via simple reactive dip-coating. J. Mater. Chem. A 5, 11009-11015 (2017).

45. Zhou, $\mathrm{H}$. et al. Highly active catalyst derived from a 3D foam of $\mathrm{Fe}\left(\mathrm{PO}_{3}\right)_{2} /$ $\mathrm{Ni}_{2} \mathrm{P}$ for extremely efficient water oxidation. Proc. Natl Acad. Sci. USA 114, 5607-5611 (2017)

46. Chen, J. Y. C., Miller, J. T., Gerken, J. B. \& Stahl, S. S. Inverse spinel $\mathrm{NiFeAlO}_{4}$ as a highly active oxygen evolution electrocatalyst: promotion of activity by a redox-inert metal ion. Energy Environ. Sci. 7, 1382-1386 (2014).

47. Carmo, M., Doubek, G., Sekol, R. C., Linardi, M. \& Taylor, A. D. Development and electrochemical studies of membrane electrode assemblies for polymer electrolyte alkaline fuel cells using FAA membrane and ionomer. J. Power Sources 230, 169-175 (2013).

48. Ellms, J. W. \& Hauser, S. J. Ortho-tolidine as a reagent for the colorimetric estimation of small quantities of free chlorine. J. Ind. Eng. Chem. 5, 915-917 (1913)

\section{Acknowledgements}

This work was funded by: the European Union's Horizon 2020 research and innovation programme under grant agreement CREATE number 721065; FEDER/Ministerio de Ciencia, Innovación y Universidades - Agencia Estatal de Investigación/RTI2018095618-B-I0; and the Generalitat de Catalunya (2017-SGR-1406 and the CERCA Programme). The authors also acknowledge BSC-RES for computational resources.

\section{Author contributions}

J.R.G.-M. and N.L. proposed the concept. F.A.G.-P. and J.R.G.-M. designed the experiments. M.B.-A. and F.A.G.-P. performed the synthesis, processing and electrochemical experiments. D.N.-C. performed the magnetic measurements and analyses. N.L. performed the computational studies. All authors wrote the manuscript.

\section{Competing interests}

The authors declare no competing interests. 